\title{
The Immune Modulation Effect of Locoregional Therapies and Its Potential Synergy with Immunotherapy in Hepatocellular Carcinoma
}

\author{
This article was published in the following Dove Press journal:
} Journal of Hepatocellular Carcinoma

\author{
Prabhsimranjot Singh ${ }^{1,2}$ \\ Sudhamshi Toom (1D ${ }^{3}$ \\ Akshay Avula ${ }^{4}$ \\ Vivek Kumar (iD ${ }^{2}$ \\ Osama E Rahma ${ }^{1,2}$ \\ 'Medical Oncology, Dana-Farber Cancer \\ Institute, Boston, MA, USA; ${ }^{2}$ Department \\ of Medicine, Brigham and Women's \\ Hospital, Boston, MA, USA; ${ }^{3}$ Hematology \\ and Oncology, Maimonides Medical \\ Center, Brooklyn, NY, USA; ${ }^{4}$ Pulmonary \\ and Critical Care, Staten Island University \\ Hospital, Staten Island, NY, USA
}

Correspondence: Prabhsimranjot Singh Email Prabhsimranjot_singh@dfci.harvard. edu

\begin{abstract}
Locoregional therapies (LRTs) including radiofrequency ablation, surgical resection, and TACE, play a pivotal role in the treatment of early stage/locally advanced hepatocellular carcinoma (HCC). Besides their direct effect on tumor cells, LRTs also play an essential role in the immunomodulation of the tumor microenvironment which is of interest in the current era of cancer immunotherapy. In this review, we describe the HCC immune microenvironment and how it is affected by LRTs as described in multiple pre-clinical and clinical studies and provide the rationale for combining LRTs with immunotherapy.
\end{abstract}

Keywords: hepatocellular carcinoma, immunotherapy, locoregional therapies

\section{Introduction}

Hepatocellular carcinoma (HCC) is the most common primary malignancy of the liver and the third leading cause of cancer related mortality in the world. ${ }^{1,2}$ The treatment options vary depending on the extent of the disease ranging from locoregional treatments (LRTs) for localized disease to systemic therapy for multifocal or metastatic HCC. $^{3}$ LRTs including radiofrequency ablation (RFA), trans-arterial chemoembolization (TACE) and cryoablation are recommended for patients who are not eligible for surgical resection or liver transplant, yet most patients eventually develop refractory disease that requires systemic therapy. ${ }^{4}$ Sorafenib has been the only FDA approved systemic therapy until recently. Many targeted therapies have shown activities in $\mathrm{HCC}$ in both the first and second-line settings and received the FDA approval including regorafenib, ${ }^{5}$ ramucirumab, ${ }^{6}$ cabozantinib $^{7}$ and Lenvatinib ${ }^{8}$ while PD-1 inhibitors (programmed cell death protein-1) including pembrolizumab and nivolumab FDA approval has been limited to the second-line setting. 9,10 The obvious question is whether there is a rationale to support the combination of immunotherapy and LRTs given the established effect of each approach in HCC and whether the modest effect of immunotherapy in the advanced setting can be moved to the adjuvant setting post LRTs. Here, we review the pre-clinical data supporting such combination and summarize the recently published and ongoing clinical studies testing the combination of LRTs and immunotherapy in HCC.

\section{The Immune Microenvironment in HCC}

There have been many attempts to classify HCC based on molecular profiling in order to determine prognosis and guide future drug discovery. Goossens el al 
described two major molecular HCC subclasses using transcriptome. The aggressive subclass contained more genetic instability, activation of known survival pathways such as MET pathway and mutation of tumor suppressor genes such as TP53. It would be interesting to study this classification relevant to immune profiling in the current era of immunotherapy. ${ }^{11}$

The liver immune microenvironment is highly complex due to its heterogeneous cellular makeup of diverse myeloid cells and lymphocytes. ${ }^{12}$ The intrinsic-immunosuppressive nature of the liver microenvironment plays a major part of barrier for anti-tumor activity. ${ }^{13} \mathrm{HCC}$ is considered an immunogenic tumor that develops in an immune-suppressed microenvironment. ${ }^{14}$ This is in part due to the inherent immune tolerability of the liver given its exposure to various antigens. ${ }^{15}$ Kupffer cells are liver macrophages that are responsible to maintain immune tolerance. They play an essential role in enhancing the immune suppressive milieu of HCC by secreting Indoleamine 2,3-dioxygenase (IDO) and IL-10 (Interleukin 10) and inhibiting the cytotoxic effect of T-cells through the PD-1 pathway. ${ }^{12,16,17}$ The HCC suppressive tumor immune microenvironment is also driven by the combination of active T-regulatory cells (T-regs) and the abundance of myeloidderived suppressor cells (MDSC). ${ }^{18-20}$ MDSCs are immature myeloid cells that exhibit their suppressive effect by inhibiting NK-cell cytotoxicity, secreting pro-inflammatory cytokines and inducing T-reg cells, all leading to further immune suppression. ${ }^{19,21}$ A recent study by Zhang et al, identified two clusters of relevant immune cells in the HCC microenvironment using RNA single-cell transcriptome analysis. A lysosome-associated membrane glycoprotein 3 positive (LAMP+) dendritic cells (DC) were defined as a mature form of dendritic cells that were the most active immune regulators of T-cells and NK cells. A strong correlation between LAMP+ DCs and T-reg cells or exhausted CD8 T cells signature was noted; implicating LAMP+ DC cells relation to T-cell dysfunction leading to immune surveillance evasion. A second subset was the tumor-associated macrophages (TAMs) that was associated with poor prognosis. These observations reflect the complex immune suppressive microenvironment in HCC and makes the case to target those immune subsets in future drug development. $^{22}$

\section{Immunotherapy in HCC (Immune Checkpoint Inhibitors)}

The indications of the PD-1 inhibitors, pembrolizumab and nivolumab, have been expanded recently to include advanced and metastatic HCC in the second-line setting post-sorafenib. The FDA approval of nivolumab and pembrolizumab was based on two clinical trials, the Checkmate -040 and Keynote-224, respectively. ${ }^{23,24}$ Both studies were singlearm Phase II open label trials enrolling 262 and 105 patients, respectively with advanced or metastatic HCC who progressed or were intolerant to sorafenib. Both studies included patients with Child Pugh A and only Checkmate 040 included patients with B7, with or without hepatitis $\mathrm{B}$ or $\mathrm{C}$. The overall objective response rate was $17 \%$ for pembrolizumab and $20 \%$ for nivolumab, with most responding patients achieving durable responses. The treatment was well tolerated across all treated cohorts whether they had or did not have viral hepatitis. Interestingly, PD-L1 (Program Cell Death Ligand 1) status did not correlate with response to nivolumab. Although a placebo controlled Phase III trial (Keynote-240) in the second-line setting showed a $20 \%$ reduction of risk of death by using pembrolizumab it failed to meet its prespecified statistical plan. ${ }^{25}$

In the front-line setting, results from recently reported randomized, phase III trial (Checkmate 459) comparing sorafenib and nivolumab did not reach pre-specified statistical significance for overall survival (OS) (HR 0.84, $\mathrm{P}=0.0419)$; although clinically meaningful improvement of OS (16.4mo vs $14.7 \mathrm{mo})$, ORR (15\% vs $7 \%$ ) and CR (4 vs 1) was noted with nivolumab vs sorafenib. Responses were noted in both PD-L1 positive and negative tumors. Grade three-fourths treatment related side effects were reported in $22 \%$ in nivolumab vs $49 \%$ in sorafenib arm. ${ }^{26}$

Other immune checkpoint inhibitors have been tested in advanced HCC with limited activity. Durvalumab is a PD-L1 antibody that demonstrated an overall response rate of $10 \%$ in pre-treated HCC. ${ }^{27}$ Tremelimumab, a CTLA-4 (Cytotoxic T lymphocyte-associated antigen 4) antibody, demonstrated similar response rate to PD-1 or PD-L1 inhibitors of $17 \%$, however, this was in a small sample of 20 patients with advanced $\mathrm{HCC}$ and chronic hepatitis $\mathrm{C}$ infection that were not candidates for surgery or LRTs. ${ }^{28}$

PD-1 or PD-L1 antibodies have been tested in combination with CTLA-4 antibodies in advanced HCC. Checkmate040 included a cohort of HCC patients with a combination of nivolumab and ipilimumab (anti-CTLA-4) in the postsorafenib setting. The response rate doubled with the combination (33\%) compared to single-agent nivolumab (although the study was not designed for such comparison) with the cost of increased rate of adverse events, mainly transaminitis and diarrhea especially in the cohort with $3 \mathrm{mg} / \mathrm{kg}$ dose of ipilimab. $^{23,29}$ The HIMALAYA Phase 3 study is currently 
assessing similar combinations of durvalumab and tremelimumab in the first-line setting compared to sorafenib. ${ }^{30,31}$

Multiple ongoing trials are evaluating combination therapy of anti-PD-1/PD-L1 or Anti-CTLA-4 antibodies with other modalities such as anti-angiogenesis. Most recently the IMbrave150 study demonstrated the combination of atezolizumab (anti-PD-L1) and bevacizumab which targets vascular endothelial growth factor A (VEGF-A) to be the first combination to increase OS and progression free survival (PFS), the co-primary endpoints of the study, compared to sorafenib in the first-line setting. After a median follow-up of 8.6 months, median OS for the atezolizumab/bevacizumab combination was not reached compared to 13.2 months for sorafenib (hazard ratio [HR] $0.58 ; 95 \%$ CI, 0.42-0.79;p $=0.0006$ ) while the median PFS of the combination was 6.8 months compared to 4.5 for sorafenib (HR 0.59 (95\% CI, 0.47-0.76; p < 0.0001). The adverse events were consistent with the safety profile of each agents on the combination arm. ${ }^{32,33}$

In summary, immune checkpoint inhibitors demonstrated a modest activity in $\mathrm{HCC}$ as single agents in the second-line setting post-sorafenib while the combination with anti-angiogenesis is moving to the front-line setting based on promising efficacy data.

\section{The Immunological Effects of Loco-Regional Therapies}

Besides local tumor control, locoregional therapies also affect tumor immunity through several interrelated but complex mechanisms. ${ }^{34}$ LRTs cause immunogenic cell death (ICD) leading to the release of various tumor antigens. ${ }^{35}$ In addition, ablation has been shown to increase dendritic cells in the HCC tumor microenvironment which leads to enhanced antigen presentation and triggers an immune response due to the activation of T-cells. ${ }^{36}$ In a preclinical study; Kaneko et al treated a xenograft mouse model implanted with an HCC cell line with RFA with or without an active variant of chemokine ligand-3 injection (CCL-3). Interestingly, a single RFA treatment inhibited the growth of contralateral non-RFA-treated tumors, by increasing $T$ cell infiltration and enhancing interferon- $\gamma$ production, leading to anti-tumor response. ${ }^{37}$ Besides activating T-cells, ablative therapies can also modulate the anti-tumor immunity through the inhibition of immune suppressive cells, most prominently the myeloid derived suppressor cells (MDSC). The RFA effect on immune response in humans has been studied using tumor samples and peripheral blood mononuclear cells (PBMCs) collected before and after RFA. ${ }^{38}$ There was a positive correlation between increased antigen-specific $\mathrm{CD} 8^{+} \mathrm{T}$ cells and decreased MDSCs after RFA and recurrence-free survival.

Similar to RFA, TACE has also been shown to play a significant role in immunomodulation through the same mechanism of ICD. ${ }^{39}$ Doxorubicin, the most commonly used chemotherapy in TACE can induce apoptosis leading to ICD and immune activation. ${ }^{40,41}$ The effect of TACE on the immune repertoire in the periphery was studied in HCC patients. While the CD4/CD8 ratio and th17 cells increased post-TACE, T-regs markedly decreased supporting a favorable immune profile post-TACE. ${ }^{42,43}$ In addition, TACE has been shown to be associated with a change in the proinflammatory cytokines with an increase of IL-6 and IL-22 in the first week after TACE which correlates with the development of hepatitis post-TACE. Interestingly, large tumors had an increase of Th-2 associated cytokines reflecting an immune-suppressive environment 2 months post-TACE. ${ }^{44}$

Cryoablation is another modality that can induce cell death and necrosis, however intracellular contents of the damaged tumor cells are preserved and can be recognized by the immune system initiating a tumor-specific immune response. ${ }^{45,46}$ Multiple studies demonstrated that, compared to RFA, cryoablation can induce more potent immune response as evidenced by elevated IL1, IL6, NF- $\kappa \beta$, and tumor necrosis factor- $\alpha$ (TNF- $\alpha){ }^{45}$ On the other hand cryoablation is shown to upregulate circulating PD-L1/PD1 which was associated with poor prognosis in HBV-related HCC. ${ }^{47}$ The induction of immune response along with the upregulation of PD-L1 represent an attractive strategy for combining cryoablation with PD-1 or PD-L1 inhibitors.

In summary, RFA, TACE and cryoablation have been shown to favorably modulate the HCC immune microenvironment which makes the case for further investigation of these modalities in combination with immune checkpoint inhibitors (Figure 1)

\section{Potential Biomarkers for Immune Response of LRTs}

Few markers have been investigated as potential biomarkers for response to LRTs. Given the mechanism of action of TACE in inducing ICD, cell death biomarkers were studied as potential predictive biomarkers including HMGB1, sRAGE, and DNase. Only elevated sRAGE preTACE and $24 \mathrm{hrs}$ after was associated with response to TACE. $^{39}$ 


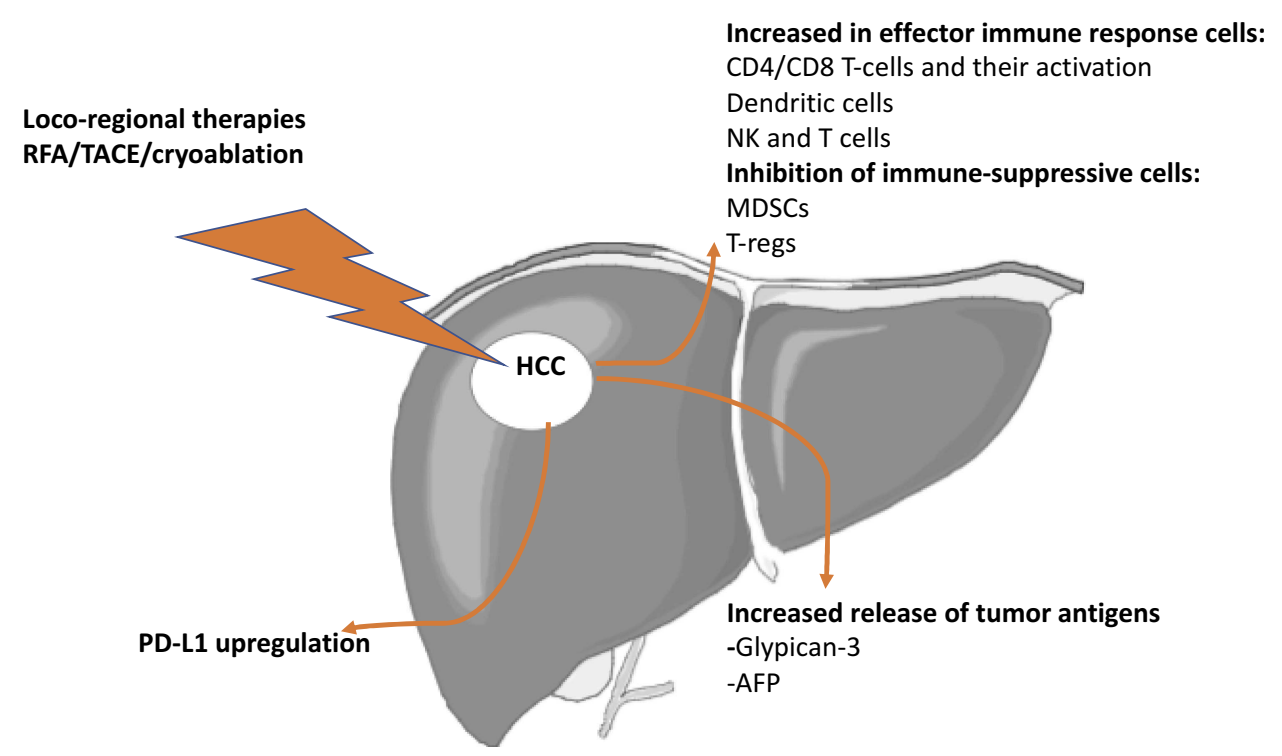

Figure I Immunological effects of Loco-regional therapies in management of HCC.

AFP ( Alpha-fetoprotein) is an oncofetal antigen that serves as a biological marker for HCC diagnosis and has been shown to correlate with response to RFA. ${ }^{48,49}$ Apart from being a diagnostic marker, AFP exhibits immune modulatory effects. AFP inhibits maturation and induce apoptosis of DC cells in addition to reduce IL-12 secretion leading to natural killer cells (NK) inhibition. ${ }^{50,51}$ Accordingly, elevated AFP may be associated with impaired immune-stimulatory effect of DC on T-cells and further studies are needed to understand the immunemodulatory effect of AFP during LRTs and immunotherapy and whether AFP could serve as a potential biomarker.

Cellular immune response to specific $\mathrm{HCC}$ associated antigens was investigated as a potential immune biomarker for RFA response. Interestingly, AFP-specific CD4 T-cells expanded after TACE and its expansion correlated with better outcomes. ${ }^{52}$ Cellular immune response against Glypican-3 (GPC3), a cell surface protein that is overexpressed in HCC, ${ }^{53}$ has been also studied in both TACE and RFA. ${ }^{54}$ Patients who underwent either RFA or TACE had a significant increase in circulating GPC3-specific cytotoxic T-lymphocytes compared to patients who underwent surgical resection which correlated with improved survival in GPC3expressing tumors. Increased Ficolin-3 expression, a recognition molecule in the lectin pathway of the complement system, in the serum post-RFA was also associated with a significant improvement in disease free survival rates. ${ }^{55}$

In summary, while many studies have proposed certain protein expression or cellular immune response against such proteins, none of these studies have been validated on a large scale and further studies are needed to identify a reliable biomarker for response to LRTs.

\section{Combing Immunotherapy and Locoregional Therapies (LRTs)}

The rationale for combining ICIs with LRTs among patients with $\mathrm{HCC}$ is based on the favorable immune modulation effects of LRTs described above that could be further enhanced by immunotherapy. In addition, RFA has been shown to increase PD-L1 expression on tumor and immune cells in patients with colorectal cancer who received RFA to their liver metastases which was possibly driven by immune activation. ${ }^{56}$ Accordingly, few studies have combined LRTs with ICIs and shown promising results. The group at NCI studied 32 HCC patients treated with a combination of LRTs with Tremelimumab (anti-CTLA4). ${ }^{57}$ The patients received a total of six doses of tremelimumab at 4-week interval followed by an intentionally incomplete RFA or DEBTACE to induce anti-tumor response at the ablation-tumor junction. Interestingly, few patients had tumor responses in untreated lesions and patients with clinical response had an increase in CD8+ T cells in tumor biopsies obtained 6 weeks post-LRTs.

In another study, Cui et al studied the combination of RFA and cellular therapy in HCC. Mononuclear cells from 30-HCC patients were harvested and induced into natural killer (NK) cells, $\gamma \delta \mathrm{T}$ cells and cytokine-induced killer (CIK) cells, which were subsequently infused back into the 
Table I Selected Ongoing Studies Using the Combination of LRTs and Immune Checkpoint Inhibitors

\begin{tabular}{|l|l|l|l|}
\hline Clinicaltrials.gov ID & Locoregional Therapy & ICI Drug & Line of IO \\
\hline NCT038I7736 & TACE and SBRT & Immune checkpoint inhibitor & Sequential use \\
NCT03638I4I & DEB (drug eluting Bead)-TACE & CTAL-4/PD-LI (Durvalumab and Tremelimumab) & Sequential use \\
NCT03I43270 & TACE & Nivolumab & Combination \\
NCT03572582 & TACE & Nivolumab & Combination \\
NCT03397654 & TACE & Pembrolizumab & Sequential \\
NCT03383458 & Ablation & Nivolumab & Adjuvant \\
NCT0282I754 & TACE, RFA, Cryo & Durvalumab, Tremelimumab & Combination \\
NCT02837029 & Yttrium Y 90 Glass Microspheres & Nivolumab & Combination \\
NCT03380I30 & Yttrium90-loaded microspheres & Nivolumab & Sequential \\
NCT03033446 & Y90-Radioembolization & Nivolumab & Combination \\
NCT03099564 & Y90-Radioembolization & Pembrolizumab & Combination \\
NCT03259867 & TATE & Nivolumab or pembrolizumab & Combination \\
NCT018536I8 & Chemoembolization (TACE)or Ablation (RFA) & Tremelimumab & Combination \\
NCT03937830 & TACE & Durvalumab also Bevacizumab & Combination \\
NCT03592706 & TACE & Immune Killer cells (IKC) & Sequential \\
NCT03575806 & TACE & Autologous Tcm Immunotherapy & Sequential \\
NCT03I24498 & TACE/RFA/PEIT & Cytokine-Induced Killer (CIK) & Adjuvant \\
NCT02568748 & TACE & Cytokine-Induced Killer (CIK & Adjuvant \\
NCT024870I7 & TACE & DC-CIK & Combination \\
NCT028568I5 & TACE & Immuncell-LC & Adjuvant \\
\hline
\end{tabular}

Note: Study status as reviewed on clinicaltrials.gov as of August 22, 2019.

Abbreviations: CIKs, cytokine-induced killer cells; DCs, dendritic cells; DEB-TACE, drug-eluting bead-TACE; PEIT, percutaneous ethanol injection therapy; RFA, radiofrequency ablation; SBRT, Stereotactic body radiation therapy; Immuncell-LC adoptive immune therapy using a CIK cell agent; TACE, trans arterial chemoembolization; TATE, trans arterial tirapazamine embolization.

RFA-treated patients for three or six courses. The combination of RFA and multiple immune cells (NK cells, $\gamma \delta$ cells and CIK cells) improved progression free survival and reduced HCC recurrence compared to RFA alone. ${ }^{58}$

While it's difficult to dissect the contribution of LRTs to immunologic response, further studies are warranted to study the benefit of adding LRTs to immunotherapy at various time intervals. A Phase I trial is underway to evaluate the safety of nivolumab with TACE using drug eluting beads (DEBTACE) (NCT03143270). Another ongoing phase II trial would evaluate the response rate of combination of TACE with nivolumab given at 1 day after vs $2-3$ days after TACE which would be repeated every 8-weeks (NCT03572582). The safety and efficacy of another PD1 inhibitor pembrolizumab is being studied in a phase I/II trial in which treatment with pembrolizumab is started 30-45 days post-TACE (using Doxorubicin and gelatin sponge)(NCT03397654). Similarly, many studies are ongoing (Table 1) to test the hypothesis of combing immune check point blockade with LRTs.

\section{Conclusion and Future Directions}

In summary, there is a strong evidence to suggest an enhanced immune modulation effect of LRTs in HCC which certainly makes the case to investigate the efficacy and potential synergy of the combination approach with immune checkpoint inhibitors. However, there are many questions that remain unanswered. What is the optimal timing of immunotherapy with regards to LRTs; is it before, during or after LRTs? What is the best biomarkers to predict response to this combination? Does PD-L1 status have any impact on response? Can immunotherapy alone cause significant immune stimulation to provide similar results in early/locally advanced HCC? The ongoing studies together with multidisciplinary collaboration may help answer some of these questions.

\section{Disclosure}

Osama E Rahma reports research support from Merck. He is a speaker for activities supported by educational grants from BMS and Merck. He is also a consultant for Merck, Celgene, Five Prime, GSK, GFK, Imvax, Defined Health INC, Roche/Genentech, Puretech, Leerink and PRMA Consulting. Dr Rahma has a patent: Methods of Using Pembrolizumab and Trebananib pending. The authors report no other conflicts of interest in this work. 


\section{References}

1. Akinyemiju T, Abera S, Ahmed M, et al. The burden of primary liver cancer and underlying etiologies from 1990 to 2015 at the global, regional, and national level: results from the global burden of disease study 2015 . JAMA Oncol. 2017;3:1683-1691. doi:10.1001/jamaoncol.2017.3055

2. Ferenci P, Fried M, Labrecque D, et al. Hepatocellular carcinoma (HCC): a global perspective. J Clin Gastroenterol. 2010;44 (4):239-245. doi:10.1097/MCG.0b013e3181d46ef2

3. (Guide) NCpGiON. Hepatobiliary cancers version 3; August 1, 2019. Accessed 27th September, 2019.

4. Medavaram S, Zhang Y. Emerging therapies in advanced hepatocellular carcinoma. Exp Hematol Oncol. 2018;7(1):1-9. doi:10.1186/ s40164-018-0109-6

5. Bruix J, Qin S, Merle P, et al. Regorafenib for patients with hepatocellular carcinoma who progressed on sorafenib treatment (RESORCE): a randomised, double-blind, placebo-controlled, phase 3 trial. Lancet. 2017;389(10064):56-66. doi:10.1016/S0140-6736(16) 32453-9

6. Zhu AX, Kang YK, Yen CJ, et al. Ramucirumab after sorafenib in patients with advanced hepatocellular carcinoma and increased $\alpha$ fetoprotein concentrations (REACH-2): a randomised, double-blind, placebo-controlled, phase 3 trial. Lancet Oncol. 2019;20(2):282-296. doi:10.1016/S1470-2045(18)30937-9

7. Abou-Alfa GK, Meyer T, Cheng AL, et al. Cabozantinib in patients with advanced and progressing hepatocellular carcinoma. $N$ Engl J Med. 2018;379(1):54-63. doi:10.1056/NEJMoa1717002

8. Kudo M, Finn RS, Qin S, et al. Lenvatinib versus sorafenib in first-line treatment of patients with unresectable hepatocellular carcinoma: a randomised phase 3 non-inferiority trial. Lancet. 2018;391 (10126):1163-1173. doi:10.1016/S0140-6736(18)30207-1

9. @US_FDA. FDA Grants Accelerated Approval to Pembrolizumab for Hepatocellular Carcinoma. FDA; 2018.

10.@US_FDA.FDA Grants Accelerated Approval to Nivolumab for HCC Previously Treated with Sorafenib. FDA; 2017.

11. Goossens N, Sun X, Hoshida Y. Molecular classification of hepatocellular carcinoma: potential therapeutic implications. Hepat Oncol. 2015;2(4):371-379. doi:10.2217/hep.15.26

12. Ringelhan M, Pfister D, O'Connor T, Pikarsky E, Heikenwalder M. The immunology of hepatocellular carcinoma. Nat Immunol. 2018;19 (3):222-232. doi:10.1038/s41590-018-0044-z

13. Jenne CN, Kubes P. Immune surveillance by the liver. Nat Immunol. 2013;14(10):996-1006. doi:10.1038/ni.2691

14. Obeid JM, Kunk PR, Zaydfudim VM, Bullock TN, Slingluff CL Jr., Rahma OE. Immunotherapy for hepatocellular carcinoma patients: is it ready for prime time? Cancer Immunol Immunother. 2018;67 (2):161-174. doi:10.1007/s00262-017-2082-z

15. Cantor HM, Dumont AE. Hepatic suppression of sensitization to antigen absorbed into the portal system. Nature. 1967;215 (5102):744-745. doi:10.1038/215744a0

16. Yan ML, Wang YD, Tian YF, Lai ZD, Yan LN. Inhibition of allogeneic T-cell response by Kupffer cells expressing indoleamine 2,3-dioxygenase. World J Gastroenterol. 2010;16(5):636-640. doi:10.3748/wjg.v16.i5.636

17. Knolle P, Schlaak J, Uhrig A, Kempf P. Meyer zum Buschenfelde KH, Gerken G. Human Kupffer cells secrete IL-10 in response to lipopolysaccharide (LPS) challenge. J Hepatol. 1995;22(2):226-229. doi:10.1016/0168-8278(95)80433-1

18. Ilkovitch D, Lopez DM. The liver is a site for tumor-induced myeloid-derived suppressor cell accumulation and immunosuppression. Cancer Res. 2009;69(13):5514-5521. doi:10.1158/0008-5472.CAN-084625

19. Hoechst B, Voigtlaender T, Ormandy L, et al. Myeloid derived suppressor cells inhibit natural killer cells in patients with hepatocellular carcinoma via the NKp30 receptor. Hepatology. 2009;50 (3):799-807. doi:10.1002/hep.23054
20. Medina-Echeverz J, Eggert T, Han M, Greten TF. Hepatic myeloid-derived suppressor cells in cancer. Cancer Immunol Immunother. 2015;64(8):931-940. doi:10.1007/s00262-015-1736-y

21. Hoechst B, Ormandy LA, Ballmaier M, et al. A new population of myeloid-derived suppressor cells in hepatocellular carcinoma patients induces $\mathrm{CD} 4(+) \mathrm{CD} 25(+) \mathrm{Foxp} 3(+) \quad \mathrm{T}$ cells. Gastroenterology. 2008;135(1):234-243. doi:10.1053/j.gastro.2008.03.020

22. Zhang Q, He Y, Luo N, et al. Landscape and dynamics of single immune cells in hepatocellular carcinoma. Cell. 2019;179(4):829845.e820. doi:10.1016/j.cell.2019.10.003

23. El-Khoueiry AB, Sangro B, Yau T, et al. Nivolumab in patients with advanced hepatocellular carcinoma (CheckMate 040): an open-label, non-comparative, Phase $1 / 2$ dose escalation and expansion trial. Lancet. 2017;389(10088):2492-2502. doi:10.1016/S0140-6736(17)31046-2

24. Zhu AX, Finn RS, Edeline J, et al. Pembrolizumab in patients with advanced hepatocellular carcinoma previously treated with sorafenib (KEYNOTE-224): a non-randomised, open-label Phase 2 trial. Lancet Oncol. 2018;19(7):940-952. doi:10.1016/S1470-2045(18)30351-6

25. Finn RS, Ryoo B-Y, Merle P, et al. Results of KEYNOTE-240: phase 3 study of pembrolizumab (Pembro) vs best supportive care (BSC) for second line therapy in advanced hepatocellular carcinoma (HCC). $J$ Clin Oncol. 2019;37(15_suppl):4004. doi:10.1200/JCO.2019.37. 15 suppl.4004

26. yau T, Park. JW, Finn RS, et al. An investigational immuno-therapy study of nivolumab compared to sorafenib as a first treatment in patients with advanced hepatocellular carcinoma. Ann Oncol. 2019. 30; Supplement 5:851-934.

27. Wainberg ZA, Segal NH, Jaeger D, et al. Safety and clinical activity of durvalumab monotherapy in patients with hepatocellular carcinoma (HCC). J Clin Oncol. 2017;35(15_suppl):4071. doi:10.1200/ JCO.2017.35.15_suppl.4071

28. Sangro B, Gomez-Martin C, de la Mata M, et al. A clinical trial of CTLA-4 blockade with tremelimumab in patients with hepatocellular carcinoma and chronic hepatitis C. J Hepatol. 2013;59(1):81-88. doi:10.1016/j.jhep.2013.02.022

29. Yau T, Kang Y-K, Kim T-Y, El-Khoueiry AB, Santoro A, Sangro B. Nivolumab (NIVO) + ipilimumab (IPI) combination therapy in patients (pts) with advanced hepatocellular carcinoma (aHCC): results from CheckMate 040. J Clin Oncol. 2019;37(15_suppl): 4012. doi:10.1200/JCO.2019.37.15_suppl.4012

30. Abou-Alfa GK, Chan SL, Furuse J, et al. A randomized, multicenter phase 3 study of durvalumab (D) and tremelimumab (T) as first-line treatment in patients with unresectable hepatocellular carcinoma (HCC): HIMALAYA study. J Clin Oncol. 2018;36(15_suppl): TPS4144-TPS4144. doi:10.1200/JCO.2018.36.15_suppl.TPS4144

31. Kelley RK, Abou-Alfa GK, Bendell JC, et al. Phase I/II study of durvalumab and tremelimumab in patients with unresectable hepatocellular carcinoma (HCC): Phase I safety and efficacy analyses. J Clin Oncol. 2017;35(15_suppl):4073. doi:10.1200/JCO.2017.35.15_suppl.4073

32. Cheng A-L, Qin S, Ikeda M, et al. IMbrave150: efficacy and safety results from a ph III study evaluating atezolizumab (atezo) + bevacizumab (bev) vs sorafenib (Sor) as first treatment (tx) for patients (pts) with unresectable hepatocellular carcinoma (HCC). Ann Oncol. 2019;30:ix186-ix187. doi:10.1093/annonc/mdz446.002

33. Finn RS, Ducreux M, Qin S, et al. IMbrave150: a randomized phase III study of $1 \mathrm{~L}$ atezolizumab plus bevacizumab vs sorafenib in locally advanced or metastatic hepatocellular carcinoma. J Clin Oncol. 2018;36(15_suppl):TPS4141. doi:10.1200/JCO.2018.36.15_suppl.TP S4141

34. Greten TF, Mauda-Havakuk M, Heinrich B, Korangy F, Wood BJ. Combined locoregional-immunotherapy for liver cancer. $J$ Hepatol. 2019;70(5):999-1007. doi:10.1016/j.jhep.2019.01.027

35. den Brok MH, Sutmuller RP, Nierkens S, et al. Efficient loading of dendritic cells following cryo and radiofrequency ablation in combination with immune modulation induces anti-tumour immunity. $\mathrm{Br}$ J Cancer. 2006;95(7):896-905. doi:10.1038/sj.bjc.6603341 
36. Dromi SA, Walsh MP, Herby S, et al. Radiofrequency ablation induces antigen-presenting cell infiltration and amplification of weak tumor-induced immunity. Radiology. 2009;251(1):58-66. doi:10.1148/radiol.2511072175

37. Iida N, Nakamoto $\mathrm{Y}, \mathrm{Baba} \mathrm{T}$, et al. Antitumor effect after radiofrequency ablation of murine hepatoma is augmented by an active variant of $\mathrm{CC}$ Chemokine ligand 3/macrophage inflammatory protein-1alpha. Cancer Res. 2010;70(16):6556-6565. doi:10.1158/ 0008-5472.CAN-10-0096

38. Mizukoshi E, Yamashita T, Arai K, et al. Enhancement of tumor-associated antigen-specific $\mathrm{T}$ cell responses by radiofrequency ablation of hepatocellular carcinoma. Hepatology. 2013;57 (4):1448-1457. doi:10.1002/hep.26153

39. Kohles N, Nagel D, Jüngst D, Stieber P, Holdenrieder S. Predictive value of immunogenic cell death biomarkers HMGB1, sRAGE, and DNase in liver cancer patients receiving transarterial chemoembolization therapy. Tumour Biol. 2012;33(6):2401-2409. doi:10.1007/ s13277-012-0504-2

40. Galluzzi L, Buqué A, Kepp O, Zitvogel L, Kroemer G. Immunological effects of conventional chemotherapy and targeted anticancer agents. Cancer Cell. 2015;28(6):690-714. doi:10.1016/j. ccell.2015.10.012

41. Kroemer G, Galluzzi L, Kepp O, Zitvogel L. Immunogenic cell death in cancer therapy. Annu Rev Immunol. 2013;31:51-72. doi:10.1146/ annurev-immunol-032712-100008

42. Liao Y, Wang B, Huang ZL, et al. Increased circulating Th17 cells after transarterial chemoembolization correlate with improved survival in stage III hepatocellular carcinoma: a prospective study. PLoS One. 2013;8(4):e60444. doi:10.1371/ journal.pone.0060444

43. Liao J, Xiao J, Zhou Y, Liu Z, Wang C. Effect of transcatheter arterial chemoembolization on cellular immune function and regulatory $\mathrm{T}$ cells in patients with hepatocellular carcinoma. Mol Med Rep. 2015;12(4):6065-6071. doi:10.3892/mmr.2015.4171

44. Kim MJ, Jang JW, Oh BS, et al. Change in inflammatory cytokine profiles after transarterial chemotherapy in patients with hepatocellular carcinoma. Cytokine. 2013;64(2):516-522. doi:10.1016/j.cyto. 2013.07.021

45. Jansen MC, van Hillegersberg R, Schoots IG, et al. Cryoablation induces greater inflammatory and coagulative responses than radiofrequency ablation or laser induced thermotherapy in a rat liver model. Surgery. 2010;147(5):686-695. doi:10.1016/j.surg.2009.10.053

46. Chu KF, Dupuy DE. Thermal ablation of tumours: biological mechanisms and advances in therapy. Nat Rev Cancer. 2014;14 (3):199-208. doi:10.1038/nrc3672
47. Zeng Z, Shi F, Zhou L, et al. Upregulation of circulating PD-L1/PD-1 is associated with poor post-cryoablation prognosis in patients with HBV-related hepatocellular carcinoma. PLoS One. 2011;6(9):e23621. doi:10.1371/journal.pone.0023621

48. He C, Zhang X, Li C, et al. Changes of alpha-fetoprotein levels could predict recurrent hepatocellular carcinoma survival after trans-arterial chemoembolization. Oncotarget. 2017;8(49):85599-8 5611. doi:10.18632/oncotarget.20343

49. Liu G, Ouyang Q, Xia F, et al. Alpha-fetoprotein response following transarterial chemoembolization indicates improved survival for intermediate-stage hepatocellular carcinoma. HPB (Oxford). 2019; 21(1):107-113. doi:10.1016/j.hpb.2018.06.1800

50. Pardee AD, Shi J, Butterfield LH. Tumor-derived $\alpha$-fetoprotein impairs the differentiation and $\mathrm{T}$ cell stimulatory activity of human dendritic cells. J Immunol. 2014;193(11):5723-5732. doi:10.4049/ jimmunol.1400725

51. Wang X, Wang Q. Alpha-fetoprotein and hepatocellular carcinoma immunity. Can J Gastroenterol Hepatol. 2018;2018:9049252. doi:10.1155/2018/9049252

52. Ayaru L, Pereira SP, Alisa A, et al. Unmasking of alpha-fetoproteinspecific CD4(+) $\mathrm{T}$ cell responses in hepatocellular carcinoma patients undergoing embolization. J Immunol. 2007;178(3):1914-19 22. doi:10.4049/jimmunol.178.3.1914

53. Wang L, Yao M, Pan LH, Qian Q, Yao DF. Glypican-3 is a biomarker and a therapeutic target of hepatocellular carcinoma. Hepatobiliary Pancreat Dis Int. 2015;14(4):361-366. doi:10.1016/S1499-3872(15)60396-4

54. Nobuoka D, Motomura Y, Shirakawa H, et al. Radiofrequency ablation for hepatocellular carcinoma induces glypican-3 peptide-specific cytotoxic T lymphocytes. Int J Oncol. 2012;40(1):63-70. doi:10.38 92/ijo.2011.1202

55. Shen S, Peng H, Wang Y, et al. Screening for immune-potentiating antigens from hepatocellular carcinoma patients after radiofrequency ablation by serum proteomic analysis. BMC Cancer. 2018;18(1):117. doi:10.1186/s12885-018-4011-8

56. Shi L, Chen L, Wu C, et al. PD-1 blockade boosts radiofrequency ablation-elicited adaptive immune responses against tumor. Clin Cancer Res. 2016;22(5):1173-1184. doi:10.1158/1078-0432.CCR-15-1352

57. Duffy AG, Ulahannan SV, Makorova-Rusher O, et al. Tremelimumab in combination with ablation in patients with advanced hepatocellular carcinoma. J Hepatol. 2017;66(3):545-551. doi:10.1016/j.jhep.2016. 10.029

58. Cui J, Wang N, Zhao H, et al. Combination of radiofrequency ablation and sequential cellular immunotherapy improves progression-free survival for patients with hepatocellular carcinoma. Int J Cancer. 2014;134 (2):342-351. doi:10.1002/ijc.28372

\section{Publish your work in this journal}

The Journal of Hepatocellular Carcinoma is an international, peerreviewed, open access journal that offers a platform for the dissemination and study of clinical, translational and basic research findings in this rapidly developing field. Development in areas including, but not limited to, epidemiology, vaccination, hepatitis therapy, pathology and molecular tumor classification and prognostication are all considered for publication. The manuscript management system is completely online and includes a very quick and fair peer-review system, which is all easy to use. Visit http://www.dovepress.com/ testimonials.php to read real quotes from published authors. 